\title{
Bragg thickness criterion for intracavity diffraction gratings
}

\author{
Ludivine Menez, Isabelle Zaquine, and Alain Maruani \\ Département Traitement du Signal et de l'Image, Ecole Nationale Supérieure de Télécommunications, \\ 46 Rue Barrault, 75634 Paris Cédex 13, France
}

\section{Robert Frey}

Départment Traitement du Signal et de l'Image, Ecole Nationale Supérieure de Télécommunications, 46 Rue Barrault, 75634 Paris Cédex 13, France, and Laboratoire Charles Fabry de l'Institut d'Optique, Institut d'Optique Théorique et Appliquée, and Centre National de la Recherche Scientifique, Bat. 503, B.P. 147, 91403 Orsay Cédex, France

Received August 7, 2001; revised manuscript received November 12, 2001

\begin{abstract}
The grating thickness limit $l_{\mathrm{FP}}$ between the Raman-Nath and the Bragg diffraction regimes is calculated for an index grating placed in an asymmetric Fabry-Perot resonator with a totally reflecting back mirror and compared with that which was obtained for the same grating with no cavity $l_{M}$. Owing to the increase of the effective interaction length inside the Fabry-Perot cavity, the stronger the front mirror reflectivity $R_{1}$ of the cavity, the smaller the thickness above which the whole diffracted intensity can be concentrated into one unique diffracted beam: $l_{\mathrm{FP}}=\left[\left(1-\sqrt{R_{1}}\right) /\left(1+\sqrt{R_{1}}\right)\right] l_{M} / 2$. (C) 2002 Optical Society of America

OCIS codes: $090.1970,090.7330,190.4360$.
\end{abstract}

\section{INTRODUCTION}

The diffraction of light on holograms is of interest for fundamental reasons and because of its applications to optical signal processing. ${ }^{1,2}$ Although Raman-Nath diffraction on thin holograms is quite useful, Bragg diffraction on thick gratings is probably more interesting because then diffracted light is condensed into one unique mode, provided that the incidence angle and wavelength of the read beam fulfill the so-called Bragg resonance condition. The transition between Raman-Nath and Bragg diffraction regimes has been studied extensively for simple gratings. ${ }^{3-8}$ The most commonly used thickness criterion is that the grating thickness be larger than some threshold, depending on the grating period and on the light's wavelength, to be in the Bragg regime where higher diffraction orders become vanishingly small.

However, even in the Bragg regime the diffraction properties of gratings are often limited by the small refractive-index changes that can be induced or by the thinness of the nonlinear materials. It has been shown $^{9-12}$ that one can overcome these limitations by putting the grating into a Fabry-Perot resonator. Strong improvement results when it is assumed that all the diffracted energy is concentrated into the main diffraction order. The diffraction regime must therefore be examined, and our aim in this paper is to derive a thickness criterion for the intracavity model.

The intensities of the higher-order diffraction modes are therefore calculated, and it is shown that, for intracavity as well as for conventional bare gratings, there is a minimum thickness above which these intensities are negligible. The model used to describe intracavity Bragg gratings is described in Section 2; all diffracted orders, even though the read beam is incident at the Bragg angle, are taken into account. The Bragg criterion is discussed in Section 3; the conventional bare grating is considered the limit where the resonator mirror reflection coefficients are zero. In Section 4 the situation of double resonance is examined, for which the main and the first higher-order diffraction modes both fulfill the Fabry-Perot resonance condition.

\section{INTRACAVITY BRAGG GRATING: THE MODEL}

A. Description of the Device

The model used for the derivation of the Bragg criterion is a direct extension of the one developed by Menez et al. ${ }^{9}$ to calculate the diffraction efficiency of intracavity Bragg gratings. In such an intracavity holographic device the lossless refractive-index grating is placed in a FabryPerot resonator; the grating fringes are parallel to the $z$ axis, whereas the cavity mirrors are parallel to the $x$ axis of our coordinate system (see Fig. 1). We consider a sinusoidal grating characterized by its average refractive in$\operatorname{dex} n_{0}$, its refractive-index modulation $\Delta n$, and its spatial period $\Lambda=2 \pi / K$ such that

$$
\begin{aligned}
n & =n_{0}+\Delta n \sin (\mathbf{K} \cdot \mathbf{r}) \\
& =n_{0}+\frac{\Delta n}{2 i}[\exp (i \mathbf{K} \cdot \mathbf{r})-\exp (-i \mathbf{K} \cdot \mathbf{r})] .
\end{aligned}
$$

The resonator has a thickness $l$ and is composed of two mirrors with amplitude reflectivities $r_{1}$ and $r_{2}$, which may be complex. 


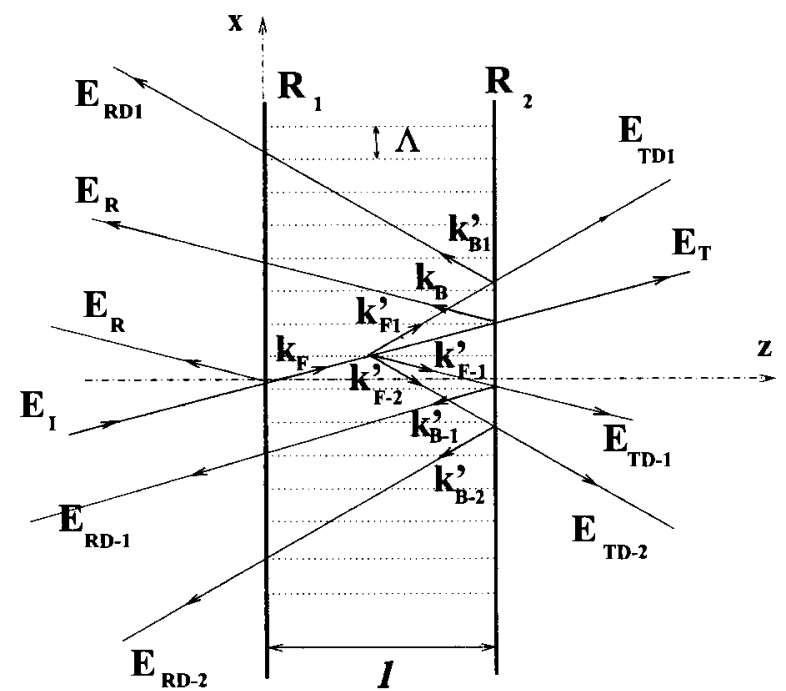

Fig. 1. Intracavity Bragg grating. The nonlinear medium where the grating is recorded is inserted into a Fabry-Perot resonator. An incident read wave $\mathbf{E}_{I}$ gives rise to reflected $\mathbf{E}_{R}$, transmitted $\mathbf{E}_{T}$, and diffracted $\mathbf{E}_{\mathrm{RD}}$ and $\mathbf{E}_{\mathrm{TD}}$ waves, the numbers in whose subscripts indicate their diffraction order. The intracavity wave vectors of the read and diffracted waves are, respectively, $\mathbf{k}$ and $\mathbf{k}^{\prime}$. They bear the subscripts $F$ for forward propagating and $B$ for backward propagating. For simplicity, refraction is not taken into account in this figure.

\section{B. Wave Equation and Phase-Matching Conditions}

An incident plane and monochromatic read wave $\mathbf{E}_{I}$ with wavelength $\lambda_{0}$ and Bragg resonant refraction angle $\theta=\arcsin (\lambda / 2 \Lambda)$ inside the cavity produces reflected and transmitted waves $\left(\mathbf{E}_{R}, \mathbf{E}_{T}\right)$ and reflected and transmitted diffracted waves $\left(\mathbf{E}_{\mathrm{RD}_{p}}\right.$ and $\left.\mathbf{E}_{\mathrm{TD}_{p}}\right)$, where $p$ is the diffraction order. The amplitudes of the forward intracavity read and $p$ th-order diffracted waves are, respectively, $R_{F}$ and $S_{F_{p}}$. Their wave vectors are $\mathbf{k}_{F}$ and $\mathbf{k}_{F_{p}{ }^{\prime}}$, respectively. The backward waves, which are due to reflection on the mirrors, have subscripts $B$ instead of $F$ (see Fig. 1). Propagation angles $\theta_{p}$ of the diffracted waves are determined by the Raman-Nath relation

$$
k\left(\sin \theta_{p}-\sin \theta\right)=p K,
$$

where $k=2 \pi / \lambda$, with $\lambda=\lambda_{0} / n_{0}$, and $K=2 \pi / \Lambda$ is also related to $\sin \theta$, which yields

$$
\sin \theta_{p}=(2 p+1) \sin \theta .
$$

The highest possible diffraction order $p_{\max }$ is limited by

$$
\left|\left(2 p_{\max }+1\right) \sin \theta\right| \leqslant 1 \text {. }
$$

This means that diffraction orders 1 and -2 can exist only for internal read angles smaller than $\theta_{\max }$ $=\arcsin (1 / 3) \simeq 19.5^{\circ}$, and the smaller the angle, the more diffraction orders in the nonlinear medium.

In the nonlinear medium the wave equation is

$$
\begin{aligned}
& \nabla R_{F} \cdot \hat{k}_{F} \exp \left(i \mathbf{k}_{F} \cdot \mathbf{r}\right)+\nabla R_{B} \cdot \hat{k}_{B} \exp \left(i \mathbf{k}_{B} \cdot \mathbf{r}\right) \\
&+ \sum_{p=0}^{p_{\max }}\left[\nabla S_{F_{p}} \cdot \hat{k}_{F_{p}}^{\prime}\right. \\
&\left.\times \exp \left(i \mathbf{k}_{F_{p}}^{\prime} \cdot \mathbf{r}\right) \nabla S_{B_{p}} \cdot \hat{k}_{B_{p}}^{\prime} \exp \left(i \mathbf{k}_{B_{p}}^{\prime} \cdot \mathbf{r}\right)\right] \\
&=\frac{2 i \pi}{\lambda_{0}}\left\{n_{0}+\frac{\Delta n}{2 i}[\exp (i \mathbf{K} \cdot \mathbf{r})-\exp (-i \mathbf{K} \cdot \mathbf{r})]\right\} \\
& \quad \times\left\{\nabla R_{F} \cdot \hat{k}_{F} \exp \left(i \mathbf{k}_{F} \cdot \mathbf{r}\right)+\nabla R_{B} \cdot \hat{k}_{B}\right. \\
& \quad \times \exp \left(i \mathbf{k}_{B} \cdot \mathbf{r}\right)+\sum_{p \neq 0}^{p_{\max }}\left[\nabla S_{F_{p}} \cdot \hat{k}_{F_{p}}^{\prime}\right. \\
&\left.\left.\quad \times \exp \left(i \mathbf{k}_{F_{p}}^{\prime} \cdot \mathbf{r}\right)+\nabla S_{B_{p}} \cdot \hat{k}_{B_{p}}^{\prime} \exp \left(i \mathbf{k}_{B_{p}}^{\prime} \cdot \mathbf{r}\right)\right]\right\},
\end{aligned}
$$

where $\hat{k}$ are unit vectors of the corresponding direction $\mathbf{k}$. Equation (5) is equivalent to a system of $2 \times\left(2 p_{\max }\right.$ +1 ) coupled equations, each corresponding to a different wave vector. The sinusoidal form of the grating is characterized by its Fourier components $\mathbf{K}$ and $-\mathbf{K}$, which means that diffraction by this grating directly couples any $p$-order mode only to $(p-1)$ - and $(p+1)$-order modes. In these conditions, only orders 1 and -1 are coupled to the read wave (order 0) and therefore give rise to high diffraction efficiencies. The higher-order modes coupled to diffracted waves are necessarily less important. To select the significant terms of each equation properly we must have a clear view of the phase-matching conditions. Figure 2 shows the various wave vectors of the RamanNath diffraction orders. The circle diameter is $k$. The Raman-Nath relation [Eq. (2)] imposes phase matching along the $x$ axis, as all the $x$ projections of the wave vectors differ from one another by an integer number of $K$, and no $x$ dependence of the amplitude is taken into ac-

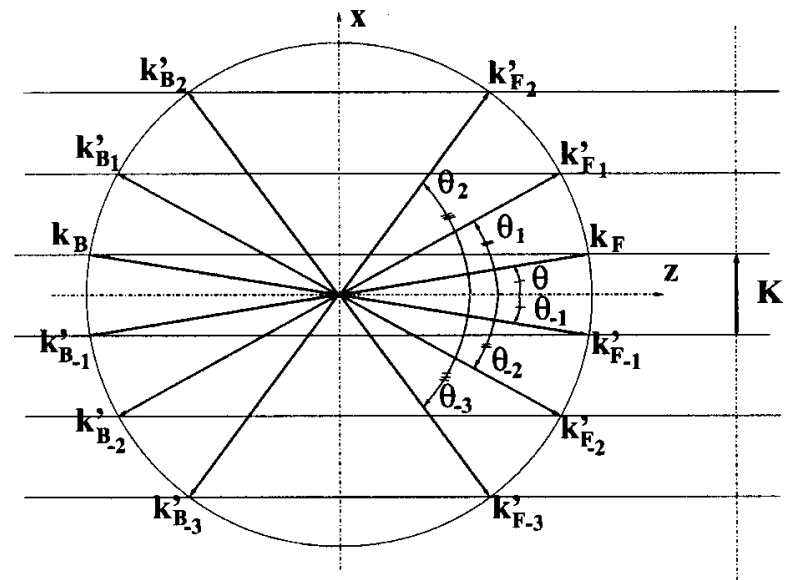

Fig. 2. Phase matching. The forward and backward wave vectors of the read wave, $\mathbf{k}_{F}$ and $\mathbf{k}_{B}$, and grating wave vector $\mathbf{K}$ are represented as well as all diffracted waves $\mathbf{k}_{F}{ }^{\prime}$ and $\mathbf{k}_{B}{ }^{\prime}$ (numbered by their diffraction orders), according to the Raman-Nath relation. 
count. $R_{F}, R_{B}$ and $S_{F_{p}}, S_{B_{p}}$ are therefore functions of $z$ only. A phase mismatch along the $z$ direction implies rapid attenuation of the corresponding wave and can be tolerated only in the case of a thin sample; the object of this study is to determine how thin that is. The -1 diffraction order is the only phase-matched mode to the forward incident wave as $\theta_{-1}=-\theta$, whereas the $|p|$ diffraction order is phase matched to the $-|p|-1$ diffraction order as $\theta_{-|p|-1}=-\theta_{|p|}$. The phase mismatch $k\left(\cos \theta_{p}\right.$ - $\left.\cos \theta_{p+1}\right) z$ increases with $|p|$; this is one more clue to help us in the simplification of Eq. (5).

We finally get two sets of four coupled propagation equations, which relate the amplitudes of order 0 ; of the main diffracted beam, order -1 ; and of the first two higher diffraction modes, order 1 and order -2 . Moreover, only the terms that have the smallest phase mismatch $\Delta k z=k\left(\cos \theta-\cos \theta_{1}\right) z=k\left(\cos \theta-\cos \theta_{-2}\right) z$ are kept in each equation:

$$
\begin{aligned}
& \cos \theta \frac{\mathrm{d} R_{F}}{\mathrm{~d} z}=\frac{\pi \Delta n}{\lambda_{0}}\left[S_{F_{-1}}-S_{F_{1}} \exp (-i \Delta k z)\right], \\
& \cos \theta \frac{\mathrm{d} S_{F_{-1}}}{\mathrm{~d} z}=\frac{\pi \Delta n}{\lambda_{0}}\left[-R_{F}+S_{F_{-2}} \exp (-i \Delta k z)\right], \\
& \cos \theta_{1} \frac{\mathrm{d} S_{F_{1}}}{\mathrm{~d} z}=\frac{\pi \Delta n}{\lambda_{0}}\left[R_{F} \exp (i \Delta k z)\right], \\
& \cos \theta_{1} \frac{\mathrm{d} S_{F_{-2}}}{\mathrm{~d} z}=\frac{\pi \Delta n}{\lambda_{0}}\left[-S_{F_{-1}} \exp (i \Delta k z)\right] \\
& -\cos \theta \frac{\mathrm{d} R_{B}}{\mathrm{~d} z}=\frac{\pi \Delta n}{\lambda_{0}}\left[S_{B_{-1}}-S_{B_{1}} \exp (i \Delta k z)\right], \\
& -\cos \theta \frac{\mathrm{d} S_{B_{-1}}}{\mathrm{~d} z}=\frac{\pi \Delta n}{\lambda_{0}}\left[-R_{B}+S_{B_{-2}} \exp (i \Delta k z)\right], \\
& -\cos \theta_{1} \frac{\mathrm{d} S_{B_{1}}}{\mathrm{~d} z}=\frac{\pi \Delta n}{\lambda_{0}}\left[R_{B} \exp (-i \Delta k z)\right], \\
& -\cos \theta_{1} \frac{\mathrm{d} S_{B_{-2}}}{\mathrm{~d} z}=\frac{\pi \Delta n}{\lambda_{0}}\left[-S_{B_{-1}} \exp (-i \Delta k z)\right] .
\end{aligned}
$$

The last step before solving these equations is to write the boundary conditions on the cavity mirrors to relate the forward-propagating waves to the backward waves on each side of the resonator $(z=0$ and $z=l$ ):

$$
\begin{aligned}
S_{F_{1}}(0) & =r_{1} S_{B_{1}}(0), \\
S_{F_{-2}}(0) & =r_{1} S_{B_{-2}}(0), \\
r_{2} S_{F_{1}}(l) & =S_{B_{1}}(l) \exp \left(-2 i k l \cos \theta_{1}\right), \\
r_{2} S_{F_{-2}}(l) & =S_{B_{-2}}(l) \exp \left(-2 i k l \cos \theta_{1}\right) .
\end{aligned}
$$

\section{Equation Resolution}

Coupled differential equations (6)-(13) cannot be simply solved analytically, and we have calculated the amplitudes of the higher-order modes in two ways.

The first one treats the system in matrix form. The unknown is a vector whose components are the four amplitudes of the relevant waves:

$$
\frac{\mathrm{d}}{\mathrm{d} z}\left(\begin{array}{c}
S_{F_{1}} \\
R_{F} \\
S_{F_{-1}} \\
S_{F_{-2}}
\end{array}\right)=[u]\left(\begin{array}{c}
S_{F_{1}} \\
R_{F} \\
S_{F_{-1}} \\
S_{F_{-2}}
\end{array}\right),
$$

where $[u]$ is a $4 \times 4$ matrix. The matrix differential equation can then be integrated by the Peano-Baker method, ${ }^{13}$ and the four modes that are considered are calculated in one step.

The second way is the method of successive approximations. ${ }^{14}$ We used the expressions of the read and -1 diffraction order waves given in Ref. 9 to calculate the higher orders added to the propagation equation at a second stage. We have not yet found any circumstances in which the two methods gave different results.

\section{THICKNESS CRITERIA}

In this section we analyze the influence of the grating length on the intensities of the various diffracted beams. The study of the intracavity device is based on the case of a simple bare grating, and the usual thickness criteria need to be reviewed before a new intracavity Bragg criterion can be derived.

\section{A. Simple Grating}

In simple gratings the Bragg regime is usually associated with thick grating diffraction, meaning that there is a single diffracted beam, ${ }^{3}$ whereas the Raman-Nath regime is understood as thin grating diffraction or the presence of multiple diffracted orders. ${ }^{4}$ The two diffraction regimes are distinguished by use of the dimensionless parameter $Q=2 \pi \lambda l / \Lambda^{2}$, where $Q<1$ for the RamanNath regime and $Q>10$ for the Bragg regime, although it is sometimes said ${ }^{7}$ that the Bragg regime starts when $Q>1$ or that, on the contrary, even for $Q>10$ the higher-order diffracted waves can be of considerable importance in gratings with large index modulation ${ }^{5}(\Delta n$ $\sim 10^{-3}-10^{-1}$ ). It is therefore necessary to be extremely precise about our own definition of the Bragg diffraction regime. We use here as a reference the criterion defined by Mallick ${ }^{8}$ : if $Q_{M}=Q / 2=\pi \lambda l / \Lambda^{2}$ is greater than 10 (or if $l>l_{M}=10 \Lambda^{2} / \pi \lambda$ ), the relative intensity of higher orders is less than 0.01. His calculation uses a constant diffracted amplitude per unit length, which cannot be extended to the intracavity case. Therefore we investigate whether his criterion remains valid when we use our model and consider the simple Bragg sample a particular case of the intracavity device for which the reflection coefficients of the mirrors are zero. It is therefore useful to recall his main results for comparison purposes. He obtained for a relative intensity of order 1

$$
I_{1} / I_{-1}=\operatorname{sinc}^{2}(\Delta k l) \simeq \operatorname{sinc}^{2}\left(Q_{M}\right)
$$




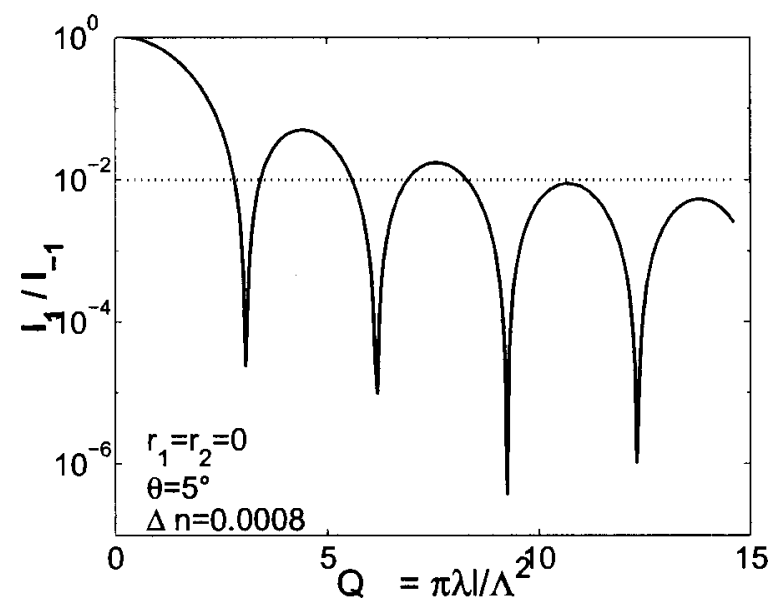

Fig. 3. Bragg criterion in a bare grating. Relative diffracted intensity $I_{1} / I_{-1}$ of order 1 normalized to order -1 is plotted versus $Q_{M}=\pi \lambda l / \Lambda^{2}$. The reference level is plotted at 0.01 (dotted line).

His Bragg criterion is based on the decrease of the maxima of the cardinal sine function, which permits definition of an absolute thickness threshold.

The result of our calculation is shown in Fig. 3. The relative diffracted intensity of order $1\left(I_{1} / I_{-1}\right)$ is plotted versus $Q_{M}$ for reflection coefficients equal to zero. Our curve is also shaped approximately as a cardinal sine. It is independent of index modulation in the range $10^{-6}$ $<\Delta n<5 \times 10^{-3}$. The agreement with the result of Mallick is good in the whole range mentioned. The highest value $\Delta n=10^{-2}$ that we have tested brings us to the limit of the validity of $Q$-type criteria, as the grating strength $^{3} \beta=\pi \Delta n l_{m} / \lambda \cos \theta$, sometimes called a modulation parameter, must then be taken into account. ${ }^{5}$ The practical range of interest for the intracavity device is restricted to the cases when a bare grating would not give a high diffraction efficiency, that is to say, when $\beta \ll 1$. This can mean a small index modulation or a limited thickness of the material, as for multiple quantum wells, which exhibit a low $\beta$ in spite of a high $\Delta n .{ }^{15,16}$ The limitation to small grating strengths therefore does not reduce the scope of our study, and we can use Mallick's formulation of the thickness criterion.

\section{B. Intensity of Diffraction Order -1}

The basic principle of our device is the choice of a resonator length such that order -1 fulfills the Fabry-Perot resonance condition. Given $\theta$, length $l_{m}$ is then a discrete parameter that is related to Fabry-Perot interference order $m$ in the following way:

$$
2 l_{m} \cos \theta=m \lambda .
$$

We use $m$ as a normalized length parameter, so our results do not depend on the wavelength or the incidence angle. When $r_{2}=1$ the whole diffracted intensity is extracted from the cavity on reflection, propagating perpendicular to the incident wave. In this way the diffracted energy is not shared between the transmitted and the reflected beams, as would be the case in a symmetric Fabry-Perot cavity. Figure 4 shows the variation of diffraction efficiency (diffracted intensity normalized to incident read intensity $I_{-1} / I_{R}$ ) versus interference order $m$ for two values of amplitude reflection coefficient $r_{1}$ of the front mirror. The curve exhibits a maximum that is close to unity for which the whole intensity of the incident wave is transferred to the reflected diffracted wave. The corresponding optimum length

$$
l_{\mathrm{opt}}=\frac{\lambda_{0} \cos \theta}{\pi \Delta n} \arccos \left(\frac{2 r_{1}}{1+r_{1}^{2}}\right)
$$

is a decreasing function of $r_{1}$ and of index modulation $\Delta n$. We are especially interested in the range of sample lengths from 0 to $l_{\text {opt }}$, where the order -1 diffracted intensity increases by several orders of magnitude toward its maximum, as there is no point in growing thicker crystals to get into a range in which the diffraction efficiency decreases.

In what follows, we examine the change caused in the intensities of orders 1 and -2 by an asymmetric FabryPerot resonator with $r_{2}=1$, which is where the intracavity device gives the best result. As the diffracted intensity is then extracted only on reflection, as mentioned above, the bare grating reference is a grating with a totally reflecting rear mirror $\left(r_{2}=1\right)$, so the diffraction properties can be directly comparable. It is interesting to note that, as far as the intensities of the diffracted beams are concerned, the bare grating with its rear mirror is actually equivalent to a double-length sample.

\section{Intensities of Diffraction Orders 1 and $\mathbf{- 2}$}

Order -2 is the mismatched result of diffraction of order -1 on the grating. In a bare Bragg grating it must remain small compared with order 1 . It was therefore not taken into account in the Bragg criterion by Mallick. Our calculations extend this result to the intracavity case. In Fig. 5 the relative intensity $I_{-2} / I_{-1}$ of order -2 is plotted versus interference order $m$ for the bare grating $\left(r_{1}=0\right)$ and for an intracavity grating $\left(r_{1}=0.9\right)$. This relative intensity is low and exhibits hardly any dependence on sample length or on reflection coefficient $r_{1}$ of the front mirror. It increases with index modulation but remains well below $10^{-2}$ as long as $\Delta n<10^{-2}$. Diffrac-

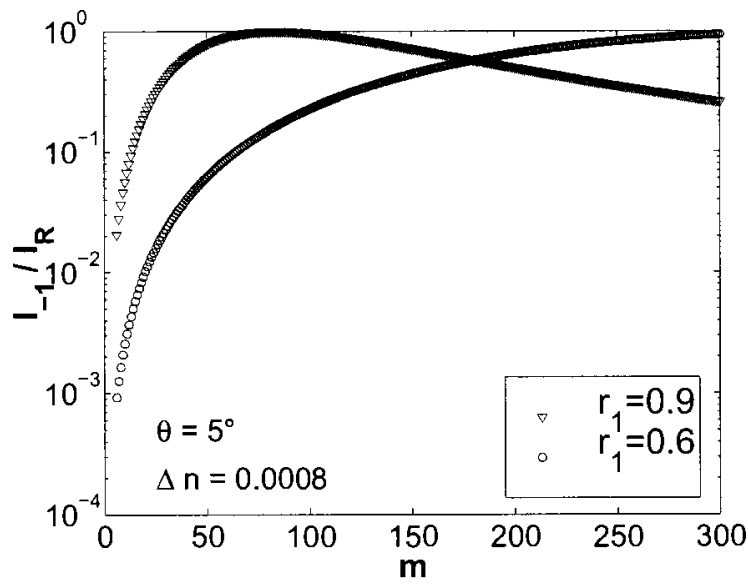

Fig. 4. Logarithmic plot of diffraction efficiency $I_{-1} / I_{R}$ of order -1 versus Fabry-Perot interference order $m$ for two reflection coefficients values, $r_{1}=0.6$ and $r_{1}=0.9$. 


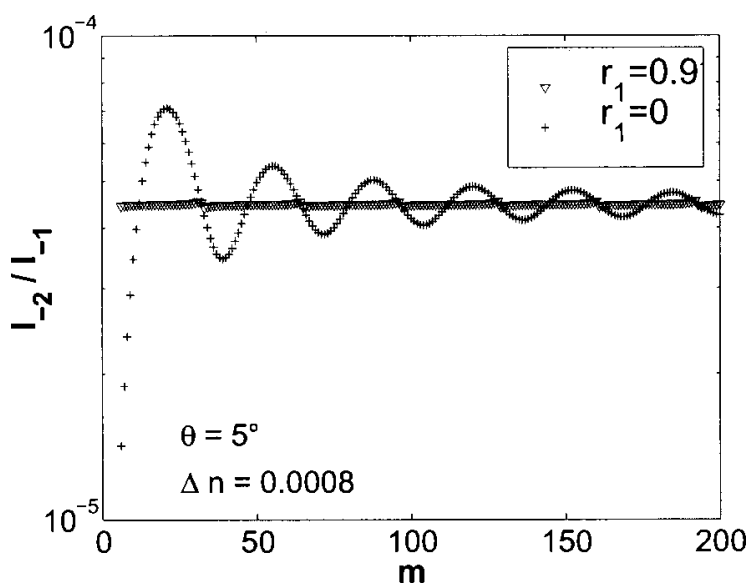

Fig. 5. Logarithmic plot of relative intensity $I_{-2} / I_{-1}$ of order -2 normalized to order -1 versus interference order $m$ for a bare grating $\left(r_{1}=0\right)$ and for an intracavity grating $\left(r_{1}=0.9\right)$.

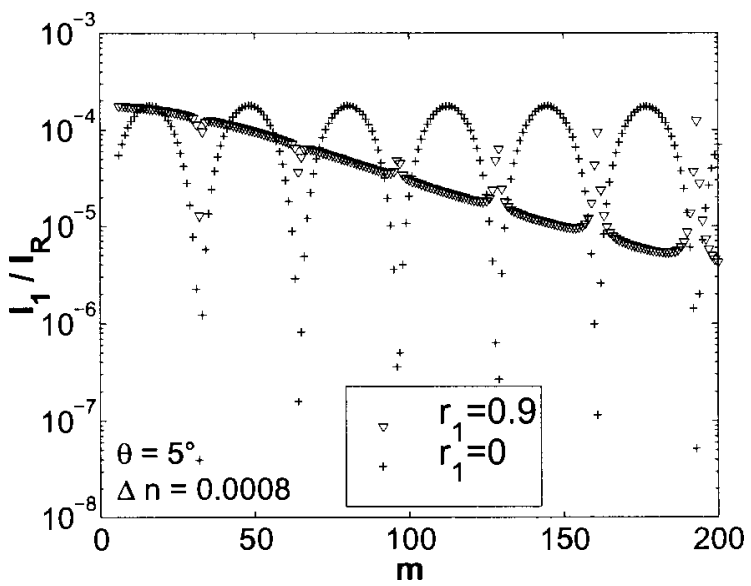

Fig. 6. Logarithmic plot of order 1 diffraction efficiency $I_{1} / I_{R}$ versus interference order $m$ for a bare grating $\left(r_{1}=0\right)$ and for an intracavity grating $\left(r_{1}=0.9\right)$.

tion order -2 can therefore be safely ignored in the derivation of the thickness threshold to the Bragg diffraction regime.

Order 1 , like order -1 , is coupled to the incident wave. The only difference between them lies in the phasematching condition, as order -1 is phase matched to the incident wave and order 1 is not. Figure 6 shows the variation of the order 1 diffraction efficiency versus $m$ for a bare grating $\left(r_{1}=0\right)$ and for an intracavity grating $\left(r_{1}=0.9\right)$. The diffraction efficiency of the bare grating is an oscillating function of $l$; the envelope of its maxima remains almost constant. The diffraction efficiency of intracavity order 1 seems to be flat compared with that of the bare grating, except for sharp peaks that correspond to the minima of the diffraction efficiency of bare grating order 1. These peaks are double-resonance peaks, as we show in Section 4 below. Anyway, it is obvious that the order 1 diffraction mode is globally attenuated by the Fabry-Perot resonator, except for its sharp maxima that nevertheless do not exceed the maxima of order 1 for a simple Bragg grating. We can therefore use Bragg order 1 as an overestimation of intracavity order 1 to define our intracavity criterion.
This preliminary study of higher-order intensities has restricted our field of investigation to the relative intensity $I_{1} / I_{-1}$ of order 1 to find out the threshold thickness from which higher-order intensities become negligible relative to that of the main diffraction order.

\section{Intracavity Bragg Criterion}

The variation of relative intensity $I_{1} / I_{-1}$ is plotted versus $m$ in Fig. 7. From our study of order 1, we know that the rapidly decreasing ratio (order $1 /$ order -1 ) is due mainly to the increasing intensity of order -1 , as the maxima of order 1 intensity do not decrease significantly. A new intracavity length criterion can then be established, assuming that the diffraction intensity of intracavity order 1 is equal to the bare sample's intensity $\left(I_{1_{\mathrm{FP}}} \simeq I_{1_{\mathrm{bare}}}\right)$, and if only the variation of intracavity diffraction order -1 is taken into account. To use Mallick's results we need to compare the ratio of order 1 to the order -1 diffracted intensities of the intracavity grating and of the bare grating. Order -1 reflected diffracted intensity $I_{-1_{\mathrm{FP}}}$ for Fabry-Perot resonant length values $l_{m}$ can be expressed analytically as a function of the bare sample diffracted intensity $I_{-1_{\text {bare }}}$ in the following way:

$$
I_{-1_{\mathrm{FP}}}=\left[\frac{\left(1-r_{1}{ }^{2}\right)}{\left(1+r_{1}{ }^{2}-2 r_{1} \cos 2 \beta\right)}\right]^{2} I_{-1_{\mathrm{bare}}} .
$$

This expression can be developed for small values of $\beta$ to give, to lowest order,

$$
I_{-1_{\mathrm{FP}}}=\left[\frac{\left(1+r_{1}\right)}{\left(1-r_{1}\right)}\right]^{2} I_{-1_{\mathrm{bare}}} .
$$

The accuracy of the development in the whole range of length that we are interested in, that is, from 0 to $l_{\text {opt }}$, depends on the value of $r_{1}$. For instance, the error is less than $1 \%$ if $r_{1}>0.82$.

From Mallick ${ }^{8}$ and taking into account that our bare sample reference with its rear mirror is equivalent to a double-length sample, we know that

$$
\text { if } \quad l>\frac{l_{M}}{2}, \quad \text { then } \quad \frac{I_{1_{\text {bare }}}}{I_{-1_{\text {bare }}}}<0.01 .
$$

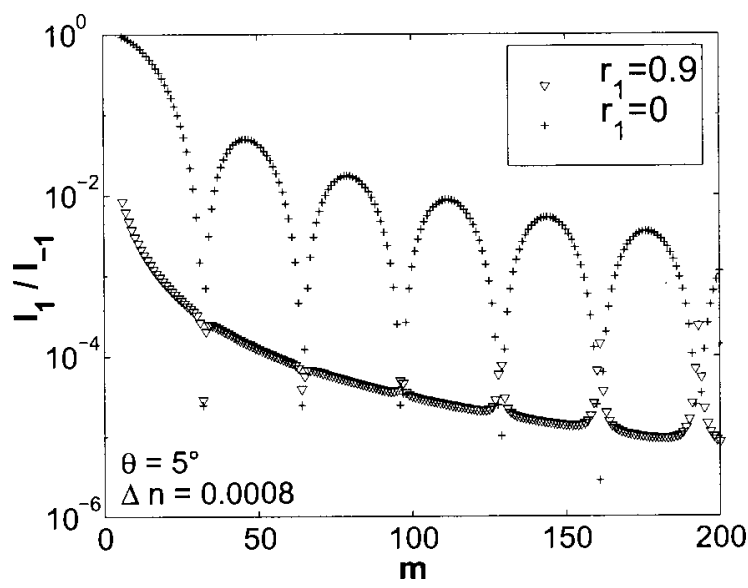

Fig. 7. Logarithmic plot of relative intensity $I_{1} / I_{-1}$ versus interference order $m$ for a bare grating $\left(r_{1}=0\right)$ and for an intracavity grating $\left(r_{1}=0.9\right)$. 
For Eq. (22) and with $I_{1_{\mathrm{FP}}}$ replaced by its overestimation $I_{1_{\text {bare }}}$,

$$
\text { if } \quad l>\frac{l_{M}}{2}, \quad \text { then } \quad \frac{I_{1_{\mathrm{FP}}}}{I_{-1_{\mathrm{FP}}}}<0.01\left[\frac{\left(1-r_{1}\right)}{\left(1+r_{1}\right)}\right]^{2} .
$$

To find the new length threshold $l_{\mathrm{FP}}$ from which the ratio $I_{1_{\mathrm{FP}}} / I_{-1_{\mathrm{FP}}}$ is smaller than $1 \%$ we use the $l$ dependence of $I_{-1_{\mathrm{FP}}}$. When we develop $I_{-1_{\text {bare }}}=\sin ^{2}(2 \beta)$ to lowest order, the $l$ dependence of $I_{-1_{\mathrm{FP}}}$ and hence of the ratio $I_{-1_{\mathrm{FP}}} / I_{1_{\mathrm{FP}}}$ appear to be purely quadratic, and, using Mallick's criterion,

$$
\text { if } \quad l>\frac{l_{M}}{2} \frac{\left(1-r_{1}\right)}{\left(1+r_{1}\right)}=l_{\mathrm{FP}}, \quad \text { then } \quad I_{1_{\mathrm{FP}}} / I_{-1_{\mathrm{FP}}}<0.01 \text {. }
$$

The lowest-order development of $I_{-1_{\mathrm{FP}}}$ can also be related to the sample length at Fabry-Perot resonance $l_{m}$ and compared to the development of the transmitted intensity diffracted by a sample with no mirror at all, $I_{-1_{\text {nomir }}}$, to introduce an effective interaction length ${ }^{10}$ that is due to the Fabry-Perot cavity:

$$
\begin{aligned}
I_{-1_{\text {nomir }}} & \sim \beta^{2}=\left(\frac{\pi \Delta n l_{m}}{\lambda \cos \theta}\right)^{2}, \\
I_{-1_{\mathrm{FP}}} & \sim\left[\frac{\left(1+r_{1}\right)}{\left(1-r_{1}\right)}\right]^{2}(2 \beta)^{2} \\
& =\left[\frac{\left(1+r_{1}\right)}{\left(1-r_{1}\right)}\right]^{2}\left(2 \frac{\pi \Delta n l_{m}}{\lambda \cos \theta}\right)^{2} \\
& =\left(\frac{\pi \Delta n l_{\mathrm{eff}}}{\lambda \cos \theta}\right)^{2}, \\
l_{\mathrm{eff}} & =\frac{2\left(1+r_{1}\right)}{\left(1-r_{1}\right)} l_{m} .
\end{aligned}
$$

To compare the intracavity Bragg criterion [inequality (25)] to the bare grating criterion [inequality (23)], we plot in Fig. 8 the relative intensity of order 1 versus the modified $Q$ parameter: $Q_{\mathrm{FP}}=\left[\left(1+r_{1}\right) /\left(1-r_{1}\right)\right] 2 Q_{M}$ for three values of the front mirror's reflectivity. It can be seen that the validity of our criterion is independent of $r_{1}$ and thus is quite general.

The Fabry-Perot resonator permits sharp attenuation of diffraction order 1 relative to length. As expected, owing to the increase of the effective interaction length, the critical length from which all diffracted energy can be considered to be concentrated in one diffracted beam is reduced further by the presence of the cavity when the reflection coefficient is large.

A numerical example of a specific sample is now given to illustrate this new criterion. We consider a grating in semi-insulating multiple quantum wells. ${ }^{15}$ The grating's thickness of $1 \mu \mathrm{m}$, grating period $\Lambda=2.4 \mu \mathrm{m}$, read wavelength $\lambda_{0}=840 \mathrm{~nm}$, and refractive index $n_{0}=3.6$ give $Q_{M}=0.12$, clearly corresponding to a Raman-Nath diffraction regime. The threshold thickness for the bare sample is $l_{M} \simeq 78 \mu \mathrm{m}$. Although the index modulation

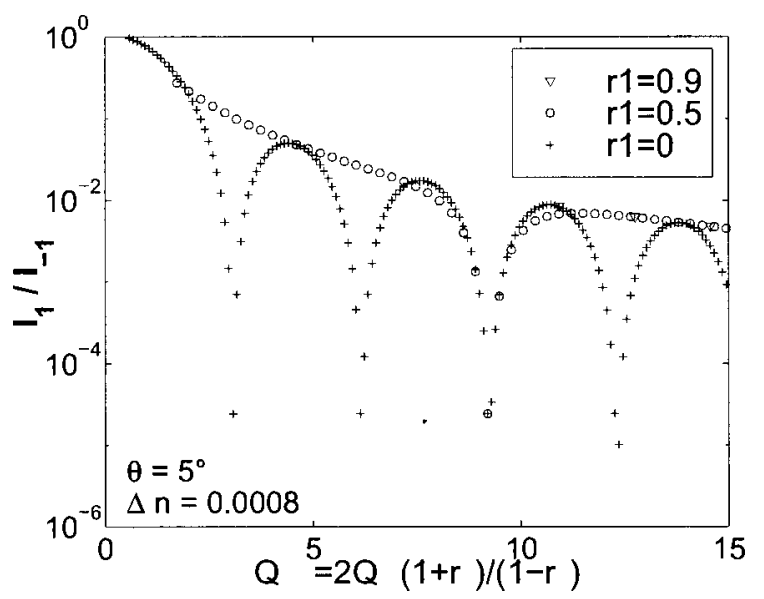

Fig. 8. Logarithmic plot of relative intensity $I_{1} / I_{-1}$ versus modified $Q$ parameter $Q_{\mathrm{FP}}=\left[2\left(1+r_{1}\right) /\left(1-r_{1}\right)\right] Q_{M}$ for three reflection coefficient values $\left(\mathbf{r}_{1}=0.9, \mathbf{r}_{1}=0.5\right.$ and $\mathbf{r}_{1}=0$, the bare grating).

is very large $\left(10^{-3}\right)$, the diffraction efficiency is only of the order of $10^{-5}$ because the sample is thin and because there are multiple diffraction beams. In an asymmetric Fabry-Perot cavity with $r_{1}=0.9$ and $r_{2}=1$ we get, using inequality (25), $l_{\mathrm{FP}} \simeq 2 \mu \mathrm{m}$, which brings the threshold down to a realistic value, even for multiple quantum wells. For the $1-\mu$ m-thick sample of Ref. 12 we get $Q_{\mathrm{FP}}$ $=4.8$, and we can see from Fig. 8 that the order 1 relative intensity has decreased to $5 \times 10^{-2}$. The diffraction efficiency can be calculated from Eq. (22), and we obtain 14\%. Absorption has not been taken into account in this derivation, although we know it can be quite large in quantum wells and would certainly reduce the diffraction efficiency of the main order. An adequate Fabry-Perot resonator nevertheless contributes to a substantial reduction of high-order diffraction and can bring thin samples into a Bragg diffraction regime.

\section{DOUBLE RESONANCE}

A reasonable idea about the effect of the Fabry-Perot cavity on orders 1 and -2 diffracted intensities is that they should be attenuated, except when they are in resonance with the Fabry-Perot cavity modes. The curves plotted in Figs. 5 and 6 confirm this guess. The attenuation of order 1 intensity increases with the resonator length, except for sharp peaks, which coincide with the minima of a simple Bragg grating that must be interpreted. It is therefore important to investigate whether orders 1 and -2 can be Fabry-Perot resonant simultaneously with order -1 . We refer to this situation as double resonance, although it is actually quadruple resonance, as it concerns the read beam, order -1 , order 1 , and order -2 $\left(\cos \theta=\cos \theta_{-1}\right.$ and $\cos \theta_{-2}=\cos \theta_{1}$ ). We show in this section how double resonance can give rise to quenching or enhancement of the diffraction efficiency of order 1 , depending on the sample length.

Three relations must be fulfilled simultaneously for double resonance to take place at a given length:

$$
\sin \theta_{1}=3 \sin \theta
$$

from the Raman-Nath relation, 


$$
2 l_{\mathrm{DR}} \cos \theta=m \lambda \quad(m \text { an integer })
$$

for the Fabry-Perot resonance condition for the read and -1 diffraction order beams, and

$$
\begin{gathered}
2 l_{\mathrm{DR}} \cos \theta_{1}=(m-\Delta m) \lambda \\
(\Delta m \text { an integer and } \Delta m<m)
\end{gathered}
$$

for the Fabry-Perot resonance condition for the 1 and -2 diffraction order beams.

It is clear that not all integer values of $m$ can be compatible with Eqs. (28)-(30), and we finally obtain

$$
\cos \theta_{\mathrm{DR}}=\left(1+\frac{\Delta m}{4 m}-\frac{\Delta m^{2}}{8 m^{2}}\right)^{-1 / 2} .
$$

In Fig. 9, angle $\theta_{\mathrm{DR}}$ is plotted as a function of the rational number $q=\Delta m / m$ with $0<q<1$. The angle increases steadily with $q$, showing a correspondence between the two parameters in the whole range of angles $\theta$ where order 1 exists: for every angle, one ratio $(\Delta \mathrm{m} / \mathrm{m})$ and hence at least one couple $(m, \Delta m)$ can be found. Double resonance of order 1 and order -1 is then possible for at least one Fabry-Perot length and all its multiples.

It is important to note that the condition for double Fabry-Perot resonance implies that the phase mismatch between the read beam and the order 1 diffraction beam is an integer number of $\pi: \Delta k l_{\mathrm{DR}}=\Delta m \pi$. We find therefore that intracavity enhancement of order 1 is neutralized because the diffracted intensity that corresponds to this length is weak. The amazing fact that we need to explain is that the double-resonance peaks of the order 1 diffraction efficiency become maxima for the higher values of $m$. Figure 10(a) is a linear plot of the order 1 diffraction efficiency, and Fig. 10(b) is a linear plot of the order -1 diffraction efficiency, both relative to interference order $m$. The juxtaposition of the two curves shows that the double resonance gives rise to minima of order 1 intensity in the range of resonator length where order -1 is increasing and to maxima in the range where order -1 is decreasing. We can compare the analytical expression obtained for the amplitude of order 1 by the successive ap-

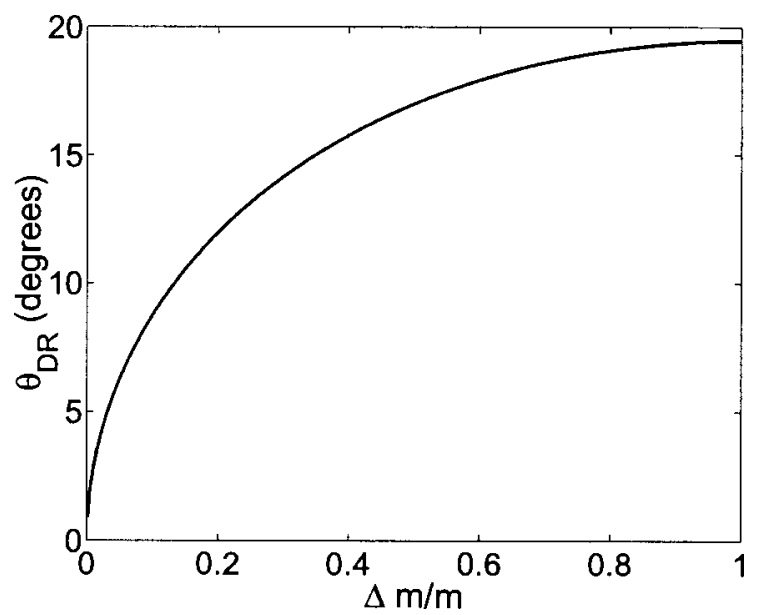

Fig. 9. Double-resonance intracavity refraction angle $\theta_{\mathrm{DR}}$ versus relative difference $\Delta m / m$ between the Fabry-Perot interference orders of diffraction orders 1 and -1 .
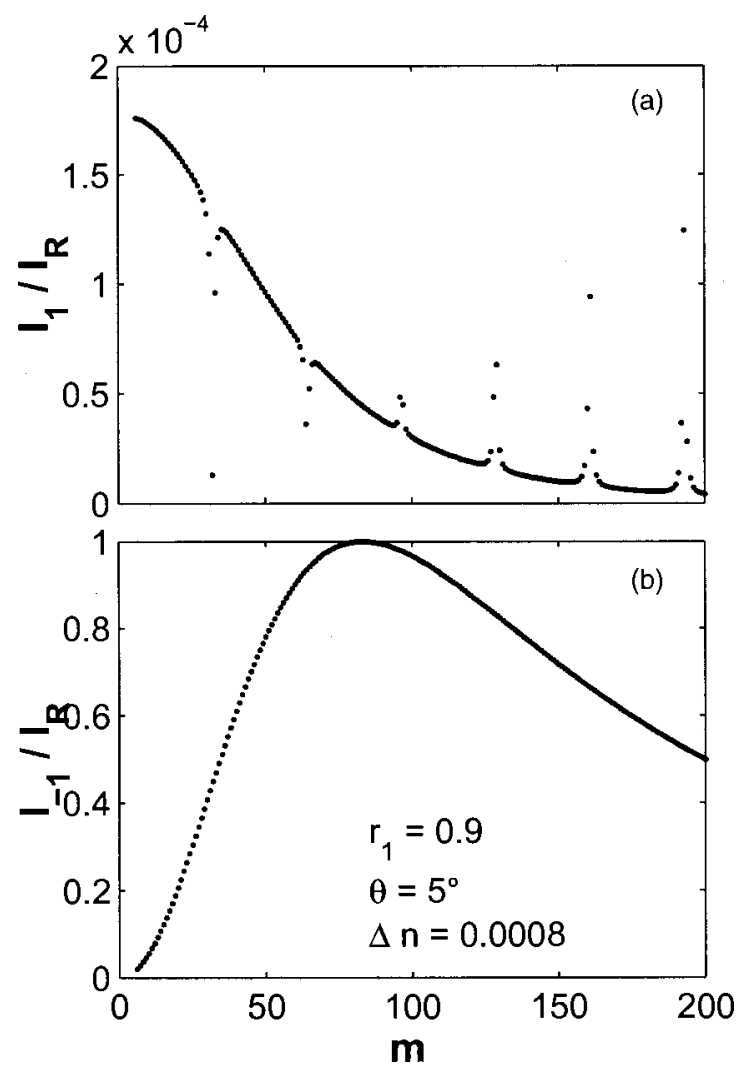

Fig. 10. Linear plots of (a) order 1 diffraction efficiency $I_{1} / I_{R}$ versus the interference order $m$ and (b) order -1 diffraction efficiency $I_{-1} / I_{R}$ versus interference order $m$.

proximation method (see Subsection 2.C) with that of the amplitude of the main diffraction order and its derivative relative to $\beta=\pi \Delta n l / \lambda_{0}$ :

$$
\begin{aligned}
S_{B_{1}}(0) \propto & \left\{\frac{-r_{F_{+}} \sin (2 \beta)+\Delta k\left[\cos (2 \beta)-r_{1}\right]}{\left(1+r_{1}^{2}-2 r_{1} \cos 2 \beta\right)}\right. \\
& \left.-\frac{\Delta k \exp \left(2 i k l \cos \theta_{1}\right)}{1-r_{1} \exp \left(2 i k l \cos \theta_{1}\right)}\right\}
\end{aligned}
$$

where $r_{F_{+}}=i \pi \Delta n / \lambda_{0} \cos \theta$ and

$$
\begin{aligned}
& S_{B_{-1}}(0) \propto \frac{\sin (2 \beta)}{\left(1+r_{1}^{2}-2 r_{1} \cos 2 \beta\right)}, \\
& \frac{\mathrm{d} S_{B_{-1}(0)}}{\mathrm{d} \beta} \propto \frac{\cos (2 \beta)\left(1+r_{1}^{2}\right) / 2-r_{1}}{\left(1+r_{1}^{2}-2 r_{1} \cos 2 \beta\right)^{2}} .
\end{aligned}
$$

The order 1 amplitude [relation (32)] is the sum of two terms. For the small values of $\beta$ considered in intracavity Bragg diffraction the first term is close to the derivative of order -1 amplitude versus $l$, especially when $r_{1}$ increases [relation (34)]. It should therefore change sign at $l_{\text {opt }}$. The second term gives rise to the double-resonance peaks when Eq. (30) is fulfilled. Its resonant value is a decreasing function of $l_{\mathrm{DR}}$ :

$$
\frac{\Delta m \pi}{\left(1-r_{1}\right) l_{\mathrm{DR}}} \text {. }
$$


The minima or maxima of the double resonance come from destructive or constructive interference between these two amplitude terms. A specific double-resonance length can hence be chosen to produce an extinction of order 1. A relative enhancement can also be obtained in the range of lengths where the main diffraction order is decreasing.

\section{CONCLUSIONS}

We have calculated the intensities of diffraction orders 1 and -2 of an intracavity grating. The intensity of order -2 is negligible, and the intensity maximum of order 1 never exceeds those of the bare grating. We have therefore been able to use Mallick's criterion to calculate a new intracavity thickness threshold, $l_{\mathrm{FP}}$. When the effective interaction length is increased, the intracavity setup of a diffraction grating can significantly reduce the thickness limit for the Bragg regime, where only one diffraction order is present. The reduction factor is an increasing function of $r_{1}$ for an asymmetric Fabry-Perot cavity: $2\left(1+r_{1}\right) /\left(1-r_{1}\right)$. The Fabry-Perot resonance of the diffraction order 1 gives rise to minima in the whole range of length from 0 to $l_{\text {opt }}$ where the main diffraction order is increasing, which definitely ensures that the Fabry-Perot grating device will widen the range of the Bragg diffraction regime. The results of this study should prove useful in the design of intracavity Bragg devices by use of thin nonlinear media such as multiple quantum wells.

I. Zaquine's e-mail address is Isabelle.Zaquine@enst.fr.

\section{REFERENCES}

1. R. J. Collier, C. B. Burckhardt, and L. H. Lin, Optical Holography (Academic, Orlando, Fla., 1971).

2. Y. Ding, D. D. Nolte, M. R. Melloch, and A. M. Weiner,
"Time-domain image processing using dynamic holography," IEEE J. Sel. Top. Quantum Electron. 4, 332-341 (1998).

3. M. G. Moharam, T. K. Gaylord, and R. Magnusson, "Criteria for Bragg regime diffraction by phase gratings," Opt. Commun. 32, 14-18 (1980).

4. M. G. Moharam, T. K. Gaylord, and R. Magnusson, "Criteria for Raman-Nath regime diffraction by phase gratings," Opt. Commun. 32, 19-23 (1980).

5. R. Magnusson and T. K. Gaylord, "Analysis of multiwave diffraction of thick gratings," J. Opt. Soc. Am. 67, 11651170 (1977).

6. T. K. Gaylord and M. G. Moharam, "Thin and thick gratings: terminology clarification," Appl. Opt. 20, 3271-3273 (1981).

7. A. Yariv and P. Yeh, Optical Waves in Crystals (Wiley, New York, 1984), Chap. 9, pp. 354-358.

8. S. Mallick, "Effets d'paisseur dans les reseaux," presented at Ecole d't d'Optolectronique Cargèse, France, summer 1990.

9. L. Menez, I. Zaquine, A. Maruani, and R. Frey, "Intracavity Bragg gratings," J. Opt. Soc. Am. B 16, 1849-1855 (1999).

10. J. H. Collet, R. Buhleier, and J. O. White, "Enhanced diffraction of light in GaAs microcavities," J. Opt. Soc. Am. B 12, 2439-2444 (1995).

11. K. M. Kwolek, M. R. Melloch, D. D. Nolte, and G. A. Brost, "Photorefractive asymmetric Fabry-Perot quantum wells transverse-field geometry," Appl. Phys. Lett. 67, 736-738 (1995).

12. D. D. Nolte, K. M. Kwolek, C. Lenox, and B. Streetman, "Dynamic holography in a broad-area optically pumped vertical GaAs microcavity," J. Opt. Soc. Am. B 18, 257-263 (2001).

13. L. A. Pipes, Applied Mathematics for Engineers and Physicists (McGraw-Hill, New York, 1958), Chap. 4, pp. 101-102.

14. M. Born and E. Wolf, Principles of Optics (Pergamon, London, 1970), Chap. 12, pp. 604-607.

15. D. D. Nolte, D. H. Olson, G. E. Doran, W. H. Knox, and A M. Glass, "Resonant photodiffractive effect in semiinsulating multiple quantum wells," J. Opt. Soc. Am. B 7, 2217-2225 (1990).

16. Q. Wang, R. M. Brubaker, D. D. Nolte, and M. R. Melloch, "Photorefractive quantum wells: transverse FranzKeldysh geometry,” J. Opt. Soc. Am. B 9, 1626-1641 (1992). 